\title{
Instrumentos para la evaluación de la calidad de vida de adultos mayores. Un estudio de revisión bibliográfica
}

\section{Instruments for the assessment of quality of life in older adults. A literature review study}

\author{
DOI: $10.46932 / \mathrm{sfjdv2n3-020}$
}

Received in: May 1st, 2021

Accepted in: Jun 30th, 2021

\author{
Juan Francisco Vélez Lucero \\ Máster en Psicoterapia \\ Psicólogo Clínico en Ministerio de Inclusión Económica y Social \\ Dirección Completa: Ecuador, Cuenca, Azuay \\ E-mail: francisco-1293@hotmail.com
}

\begin{abstract}
RESUMEN
Introducción: Estudiar la calidad de vida de las personas adultas mayores aporta estadísticamente al desarrollo biopsicosocial en Latinoamérica; al mismo tiempo, permite evaluar la eficacia de los servicios públicos/privados, contexto económico, sociocultural y familiar. Objetivo: Realizar una revisión reflexiva de instrumentos que valoran la calidad de vida de las personas adultas mayores; mediante la abstracción de las investigaciones publicadas en los repositorios digitales. Método: A través de la revisión bibliográfica de estudios científicos, exclusivamente de población adulta mayor, que validan los instrumentos en base a su consistencia interna; en idioma inglés, portugués y español. Seis repositorios digitales fueron elegidos para realizar la búsqueda: Scopus, Web of Science, PubMed, Springer, ResearchGate y Scholar Google. Resultados: Se establecieron cinco instrumentos que demostraron tener respaldo científico y aplicabilidad en personas adultas mayores, cuestionario WHOQOL-BREF, cuestionario WHOQOL-OLD, cuestionario OPQOL-35, cuestionario SODA y cuestionario WOOP. Conclusiones: Los resultados mostraron que el WHOQOL-OLD y WHOQOL-BREF son útiles en pacientes clínicos, institucionalizados, con prevalencia de enfermedades y personas adultas mayores en general. Entre otros hallazgos, los cuestionarios (OPQOL-35, SODA y WOOP) son relativamente actuales, pero expusieron grandes aportes al establecer nuevas dimensiones de satisfacción personal que se encuentran relacionados con la calidad de vida en la población adulta mayor.
\end{abstract}

Palabras Clave: "quality of life", "older people", "psychometric properties", "psychology".

\begin{abstract}
Introduction: Studying the quality of life of older adults contributes statistically to biopsychosocial development in Latin America. At the same time, it allows evaluating the effectiveness of public/private services, economic, socio-cultural, and family context. Aim: Reviewing instruments that assess the quality of life of older adults; by abstracting the research published in digital repositories. Method: Doing some reviewing of the bibliography of some scientific studies, focusing exclusively on the elderly population, which validate the instruments based on their internal consistency; in English, Portuguese and Spanish. There were six digital repositories for the search which helped the research be sustainable: Scopus, Web of Science, PubMed, Springer, ResearchGate, and Scholar Google. Results: Five evaluating instruments showed to have scientific support and applicability in older adults. They were: a WHOQOL-BREF survey, a WHOQOL-OLD survey, OPQOL-35 survey, SODA survey, and WOOP survey. Conclusions: These conclusions have shown that the WHOQOL-OLD and WHOQOL-BREF are helpful in clinical patients, with a tendency of diseases and older adults in general. Among other results, the surveys (OPQOL-35,
\end{abstract}


SODA, and WOOP) are relatively current; nonetheless, they made acceptable contributions by establishing new dimensions of personal satisfaction that relate to the quality of life in the elderly population.

Keywords: "quality of life", "older people", "psychometric properties", "psychology".

\section{INTRODUCCIÓN}

Debido a la evolución demográfica, la población de personas adultas mayores incrementará hasta 2.000 millones en los próximos 30 años (WHO, 2015); esto sin duda generará un impacto en la calidad de vida de este grupo etario debido al aumento en la prevalencia de enfermedades crónicas degenerativas no trasmisibles (Varela, 2016) y de estudios clínicos, avances en atención sanitaria, evaluaciones económicas o encuestas sobre la salud de la población (Flores-Herrera et al., 2018).

La calidad de vida es subjetiva y comprende todos los factores relacionados a satisfacer las necesidades que presentan los seres humanos a lo largo de su ciclo vital, se encuentran estrechamente vinculados al ambiente socio-cultural, características sociodemográficas, enfermedades crónicas, estado funcional, red de apoyo o el entorno (Machón et al., 2017; Power et al., 1999). La persona adulta mayor percibe como satisfactoria su calidad de vida cuando mantiene su funcionalidad física y mental (Reyes Rodríguez, \& Durand Rill, 2018); por lo tanto, es importante para los profesionales de salud, estudiantes e investigadores contar con un indicador al momento de evaluar la calidad de vida de las personas adultas mayores y de esta manera mejorar los servicios de atención preventiva, curativa y de rehabilitación a nivel personal o general. El plan de vida de las personas adultas mayores debe garantizar el envejecimiento activo y saludable, la independencia en sus actividades de la vida diaria y la satisfacción de sus necesidades básicas; además de capital social, inclusión sociocultural, actividades de ayuda mutua, etc. (Nuntaboot, Boonsawasdgulchai, \& Bubpa, 2019; Callaghan, Brookes, \& Palmer, 2017).

En la actualidad, existen varios instrumentos que evalúan la calidad de vida, se diferencian según el contexto de aplicación, aspectos culturales y dialécticos, edad del grupo poblacional, grado de deterioro físico o cognitivo, etc., y depende del evaluador seleccionar el más adecuado al momento de establecer su objetivo y población de estudio.

El cuestionario de Calidad de Vida de la Organización Mundial de la Salud en su versión corta (WHOQOL-BREF) fue desarrollado por el "WHOQOL Group" en el año de 1993, con la finalidad de medir la percepción de la persona, con o sin enfermedad, sobre su calidad de vida en general (global) y la satisfacción percibida en relación a su salud física, salud psicológica, relaciones sociales y el ambiente; su versión al español fue aprobada en el año de 1998, por el mismo grupo, su versión en sueco fue utilizada en una investigación con 254 familias de adultos mayores que viven en residencias, demostrando ser fiable 
y con buena consistencia interna (Rosén, Ahlström \& Lexén, 2020), existen otros estudios encontrados en versión pakistaní (Saquib-Lodhi et al., 2017), inglés, chino y malayo (Cheung et al., 2017), amárico (Reba, Birhane \& Gutema, 2019), taiwanés (Lin et al., 2019). Para la validación del WHOQOL-BREF se aplicó la prueba a población en general, pacientes ambulatorios, pacientes con esquizofrenia y a sus cuidadores (Lucas, 1998); además, existen estudios que ilustran la validez del instrumento, aplicándolo a una muestra de 250 personas adultas mayores, quienes reflejaron satisfacción en las cuatro áreas específicas que mide la prueba, los resultados fueron: $62.72 \%$ para salud física, $62.78 \%$ para salud psicológica, $63.89 \%$ para relaciones interpersonales y 63.29\% para el ambiente (Robles, 2017); en otro estudio, se evidenció una satisfacción global de $54.45 \%$ de los participantes y para el cálculo de las dimensiones se alcanzaron medias porcentuales de 39.32\%, 42.67\%, 39.30\% y 37.40\% respectivamente (Flores-Herrera et al., 2018).

Por otra parte, para la versión del Cuestionario de Calidad de Vida para Adultos Mayores (WHOQOL-OLD) se utilizó una muestra total de 7.400 personas alrededor del mundo quienes formaron parte de un plan piloto, patrocinado por la OMS, para validar esta nueva escala específicamente dirigida a la población adulta mayor, resultando ser un instrumento eficaz que mide la satisfacción global, así como, la calidad percibida dentro de 6 dimensiones específicas: habilidades sensoriales, autonomía, actividades pasadas, presentes y futuras, participación social, muerte e intimidad; su versión final fue establecida en el año 2005 (Power, Quinn \& Schmidt, 2005), su versión en portugués fue publicado en el año 2006 (Fleck, Chachamovich \& Trentini, 2006), su versión en español a partir del año 2011 (LucasCarrasco, Laidlaw \& Power, 2011) y se ha encontrado publicaciones del instrumento adaptado a idiomas como: Holandés, Alemán, Noruego, Francés, Turco, Coreano, Chino (Gobbens \& van Assen, 2016); a su vez, investigaciones realizadas con el cuestionario WHOQOL-OLD validan la eficacia del instrumento pues ha sido utilizado para identificar las dimensiones que presentan mayor impacto en esta población; los resultados de una muestra de 921 participantes, mostraron utilidad para diferenciar en grupos a los adultos mayores que son sanos de aquellos que tienen patologías y síntomas de depresión leves y severas, de igual manera, su consistencia interna y su validez convergente como divergente demostraron ser excelentes (Vilar, Sousa \& Simões, 2016); 903 participantes proporcionaron como resultado puntuaciones significativas en las dimensiones de autonomía, actividades pasadas, presentes y futuras, participación social e intimidad (Hussenoeder et al., 2020); 1.691 personas adultas mayores reflejaron significancia en los dominios de relaciones sociales y muerte (Tavares et al., 2016).

Del mismo modo, en el año 2008 Ann Bowling e investigadores de la universidad de Gran Bretaña desarrollaron el cuestionario denominado: "Calidad de Vida de las Personas Mayores" (OPQOL-35) en su idioma original, en el cual se empleó una muestra de 987 adultos mayores ingleses de más de 65 años, para sustentar su hipótesis que describe a la calidad de vida como un constructo multifactorial (Bowling 
\& Rai, 2009; Mares, Cigler \& Vachkova, 2016), posteriormente se publica su versión corta OPQOL-Bref en el 2013 que consta de 13 ítems considerados los más importantes por los encuestados, descartando 22 ítems del cuestionario original, demostrando ser una herramienta útil cuando se requiere una evaluación corta e implacable (Bowling et al., 2013), de igual manera se han realizado investigaciones para validar las versiones traducidas del cuestionario; es el caso de una muestra de 478 participantes, quienes reflejaron niveles altos de satisfacción en su calidad de vida, en siete dominios principales, quienes mantienen autonomía en sus actividades diarias y viven solo o con pareja, a diferencia de aquellos que se encuentran bajo el cuidado de residencias, tutores, familiares; la validez y confiablidad en esta investigación osciló entre 0.72 y 0.90 para su versión en checo (Mares, Cigler \& Vachkova, 2016), referente a su versión reducida que consta de 7 dimensiones, se realizó la validación en 168 participantes para su versión en turco, donde se encontró que el cuestionario es rentable y cuenta con una buena consistencia interna para el resultado de sus subescalas (Caliskan et al., 2019), 521 personas adultas mayores que viven solas participaron en un estudio que utilizó la versión adaptada al idioma chino, presentando resultados confiables en las 8 dimensiones y ubicándolo como instrumento idóneo para evaluar la calidad de vida en adultos mayores que viven solos (Chen \& While, 2019), en otro contexto sociocultural participaron 764 adultos mayores quienes respondieron el cuestionario en su versión e idioma original, reflejando que la calidad de vida está estrechamente relacionado a una buena salud física y emocional, independencia, toma de decisiones y control sobre su vida (Siriwardhana et al., 2019), en su versión persa los resultados de 500 y 525 participantes demostraron su utilidad para evaluar la calidad de vida en edades avanzadas para población de habla persa (Nikkhah et al., 2018; Feizi \& Heidari, 2020).

Cabe mencionar que en la actualidad se han realizado revisiones sistemáticas acerca de instrumentos idóneos para medir la calidad de vida y el bienestar de las personas adultas mayores, de las cuales resulta un nuevo cuestionario denominado SODdisfazione dell'Anziano-SODA (Martín-María et al., 2020), diseñado para medir el bienestar mental del adulto mayor, su origen es italiano, y fue sometido a validación en el año 2019 por un grupo de investigadores, en el cual participaron 135 adultos jóvenes dentro de un plan piloto y en un segundo estudio 474 adultos mayores que respondieron al SODA, verificando que el cuestionario es un instrumento breve, pero fiable para evaluar la satisfacción personal de la persona adulta mayor (Fastame, Penna \& Hitchcott, 2019), argumentando ser un instrumento que cuenta con altas propiedades psicométricas, consistencia interna y calidad de evidencia alta (Martín-María et al., 2021).

Similar ocurre con el cuestionario de Bienestar de Personas Mayores (WOOP), que fue desarrollado a partir de un estudio previo, del cual se recogieron 9 dimensiones que se relacionan con el bienestar percibido de las personas adultas mayores (Hackert et al., 2019), sujeto a validación por primera 
vez en los años 2017-2018, su versión original se encuentra en lenguaje holandés y traducida al inglés, durante el estudio se realizaron dos etapas en las que participaron un total de 1382 adultos mayores (Hackert, van Exel \& Brouwer, 2020), resultando ser un instrumento que capta adecuadamente los aspectos relacionados al bienestar de las personas adultas mayores.

Es notable la necesidad de contar con instrumentos satisfactorios al momento de evaluar la calidad de vida de personas adultas mayores en Latinoamérica, que sea de fácil aplicación, útil en diversos contextos como el área de salud, social y de investigación, adaptado a la cultura de estudio, elegido por profesionales dedicados a la atención, prevención, tratamiento y rehabilitación de patologías físicas y mentales, estudiantes, investigadores y especialmente cuando el profesional de la salud mental requiera una herramienta confiable para valorar al paciente y planificar su intervención psicoterapéutica; de esta manera se potencia la rehabilitación a través de estrategias y aptitudes que fortalecen el envejecimiento activo y saludable de las personas adultas mayores, puesto que, las políticas públicas que trabajan y protegen a este grupo etario se va reforzando cada vez más a nivel mundial y por tal razón, surge la importancia de investigar la percepción de calidad de vida que tienen las personas adultas mayores con la finalidad de contextualizar su función actual y fortalecer su potencial.

Estudiar la calidad de vida en personas adultas mayores es importante pues aporta estadísticas reales de las condiciones de vida en los diferentes contextos de Latinoamérica, mejora el estilo y la eficacia de los servicios públicos y privados, brinda estrategias de intervención tanto físico, psicológico, social, familiar, cultural e institucional para la población en general; cada vez es más real la idea de que la población envejece y con ello los problemas biopsicosociales y económicos, evaluar la calidad de vida en un contexto actual, debido a que los recursos que se disponen en la actualidad son diferentes a los que se mantenían en siglos pasados, por ello nace la importancia de contar con datos y estadísticas actualizadas en Latinoamérica para impulsar a los estados a generar estrategias de acción cuando, en un futuro, la población lo requiera; pues se pronostica que para el año 2050 se multiplicarán las personas de más de 85 años que tengan un grado moderado - alto de dependencia en sus actividades de la vida diaria (WHO, 2015). A nivel clínico, el aporte de contar con una herramienta psicométrica de alto valor que sea utilizada de manera general y que evalúe la calidad de vida de manera multifactorial, exclusiva para población adulta mayor, con la finalidad de proporcionar ventajas cuando se pretenda interpretar los resultados obtenidos, evitar la distorsión de datos y en un gran nivel, facilitar la labor interdisciplinaria de los profesionales de la salud y de los procesos psicoterapéuticos.

Por todo lo antes expuesto, el objetivo para este estudio es realizar una revisión reflexiva de instrumentos que valoran la calidad de vida de las personas adultas mayores; mediante la abstracción de las investigaciones publicadas en los repositorios digitales. 
Una vez obtenidos los resultados, se pretende consagrar un instrumento idóneo que cuente con bases científicas y que haya sido puesto a prueba para validar su consistencia interna.

\section{METODOLOGÍA}

El presente artículo se realizó mediante un diseño de revisión bibliográfica de investigaciones recopiladas de estudios científicos que validan los instrumentos, a través de su consistencia interna, utilizados a lo largo del mundo para medir la calidad de vida exclusivamente de personas adultas mayores, en idioma inglés, portugués y español, publicados dentro de un rango de 5 años previos a la fecha actual con la finalidad de evidenciar su actualidad. Seis repositorios digitales fueron elegidos para realizar la búsqueda: Scopus, Web of Science, PubMed, Springer, ResearchGate y Scholar Google. Para facilitar la búsqueda se incluyeron palabras claves como: "quality of life", "older people", "psychometric properties", "psychology". Se integró el descriptor booleano "and" para complementar la búsqueda.

Los criterios de inclusión que se aceptaron para el presente artículo fueron: a) estudios descriptivos, cuantitativos, longitudinales, transversales, correlaciones, comparativos, de revisión sistemática, b) participantes: adultos mayores de más de 60 años, c) periodo de 5 últimos años con estudios a nivel mundial, d) publicaciones en inglés, portugués y español. Por otra parte, los criterios de exclusión fueron: a) estudios cualitativos, b) estudios de caso, c) intervenciones psicoterapéuticas, d) tesis de grado y posgrado, e) participantes: menores de edad.

\section{RESULTADOS}

Acorde a la búsqueda de artículos científicos se incluyeron cincuenta y cinco para completar la presente revisión, posterior a ellos se establecieron cinco categorías; cuestionario whoqol-bref, cuestionario whoqol-old, cuestionario opqol-35, cuestionario soda y cuestionario woop.

\section{Cuestionario WHOQOL-BREF}

Las propiedades psicométricas del WHOQOL-BREF en su versión al español demostraron una consistencia interna de 0.70 para sus dimensiones y 0.89 de manera global, una correlación entre sus factores que alcanzaron los 0.91, obtenido en una muestra de 678 participantes (Huerta, Romo \& Tayabas, 2017), de igual manera, en su escala total se evidenció una puntación 0.90 de consistencia interna a partir de una encuesta a 286 adultos mayores españoles (Lucas-Carrasco, Laidlaw \& Power, 2011), 0.88 de consistencia interna del instrumento de su versión en lenguaje español y puntajes que oscilan entre 0.70 y 0.79 para cada dimensión en una muestra de 1520 adultos mayores mexicanos (Espinoza et al., 2011); de igual manera, se evidenciaron puntuaciones alpha de Cronbach entre $0.78-0.82$ para en un estudio realizado a 3400 adultos asiáticos hasta los 95 años (Suárez, Tay \& Abdullah, 2018). En lo que concierne 
a la utilidad y eficacia de esta prueba, se evidenció que, en centros de atención sanitaria, pacientes clínicos/hospitalizados e instituciones relacionadas a actividades de cuidado físico-mental del adulto mayor y en general, emplean el cuestionario WHOQOL-BREF para evaluar la calidad de vida de las personas adultas mayores (Samy et al., 2020; Orellana, Manthorpe \& Tinker, 2020; Kruithof et al., 2018; Snell et al., 2016).

\section{Cuestionario WHOQOL-OLD}

En un estudio comparativo realizado en Brasil con 600 personas adultas mayores se evidenció que la fiabilidad del WHOQOL-OLD en su versión original oscilaba entre 0.50 a 0.70 y en su versión reducida oscilaba de 0.52 a 0.77 presentando un mejor ajuste y menor probabilidad de error (De Melo et al., 2018), dentro de un estudio con 120 adultos mayores mexicanos determinaron que el alpha de Cronbach para la versión en español fue de 0.89 y para una muestra de 794 personas de 60 a 89 años, su consistencia interna total alcanzó los 0.84 , demostrando ser una herramienta útil dentro de población hispanohablante (Hernández-Navor et al., 2015; Quiroz et al., 2013), en otro estudio con 921 adultos mayores de Portugal la consistencia interna del WHOQOL-OLD tanto en su versión original como en la versión portuguesa europea demostraron excelentes parámetros de fiabilidad y validez (Vilar, Sousa \& Simões, 2016); centros de salud pública, residencias de ancianos e investigadores utilizan esta herramienta para medir la calidad de vida de sus adultos mayores en diferentes escenarios y culturas (Bangun, Gondodiputro \& Andayani, 2020; Leles da Costa Dias et al., 2019; Amonkar et al., 2018).

\section{Cuestionario OPQOL-35}

El cuestionario de la calidad de vida para las personas mayores (OPQOL-35) en su versión original reflejó una consistencia interna entre 0.70 a 0.90 de sus ocho dimensiones, siendo un instrumento útil para medir la calidad de vida de la población adulta mayor en un entorno multifactorial (Nikkhah et al., 2018); para su versión reducida que consta de 8 ítems, resulta ser un instrumento válido y confiable cuando se trata de adultos mayores que se encuentran en centro residenciales (Haugan et al., 2020), es utilizado en contextos urbanos y rurales para comparar los factores relacionados a la calidad de vida de la población adulta mayor (Siriwardhana et al., 2019).

A continuación, se presenta la revisión de las nuevas actualizaciones encontradas en los repositorios digitales, relativas al análisis de la calidad de vida de las personas adultas mayores.

\section{Cuestionario SODA}

Refiriéndose a nuevas escalas para medir la calidad de vida, se encuentra el SODA, cuestionario de satisfacción personal de ancianos, que refiere una puntuación de 0.77 de consistencia interna general (Fastame, Penna \& Hitchcott, 2019).

\section{Cuestionario WOOP}


La fiabilidad para este cuestionario fue de 0.89 en las puntuaciones de test-retest, registrando alta validez frente a la evaluación económica como al bienestar relacionado con la atención sanitaria (Hackert, van Exel \& Brouwer, 2020).

\section{DISCUSIÓN}

La versión breve del cuestionario de calidad de vida de la Organización Mundial de la Salud demuestra tener puntuaciones altas en la consistencia interna y ser una herramienta útil para pacientes clínicos desde los 18 años en adelante (Snell et al., 2016; Perera et al., 2018), además, ha demostrado una alta validez y fiabilidad en evaluaciones correspondientes a la dimensión biológica, psicológica y ambiental en pacientes hospitalizados (Kruithof et al., 2018). En lo que concierne a estudios transculturales, promoción y prevención de salud, desarrollo de servicios relacionados a la calidad de vida y epidemiología de la población adulta mayor, el módulo WHOQOL-OLD, evalúa el impacto de los servicios de atención sanitaria, acción social, economía, ambiente, etc., que son accesibles para la población y a su vez, el nivel de satisfacción que el evaluado percibe y cómo influye en el envejecimiento activo y saludable (Power et al., 1999). Estudios actuales cuentan con evidencias de una alta consistencia interna del cuestionario WHOQOL-OLD (Rezaeipandari et al., 2020 \& Kim et al., 2021). Los cuestionarios WHOQOL en sus versiones Brief y Old son utilizados especialmente en aquellos adultos mayores que acuden a centros residenciales y a espacios de socialización cómo son los centros diurnos (Simeão et al., 2018). De otro estudio se concluyó que los dos cuestionarios pueden ser aplicados de manera virtual en población adulta mayor, pues cuentan con buena consistencia interna y aceptación (Casamali et al., 2019).

El cuestionario OPQOL en comparación con el WHOQOL-OLD, demostró ser un instrumento psicométrico con fiabilidad y validez aceptable para medir la calidad de vida de personas adultas mayores que no se encuentran institucionalizados y de diversidad étnica (Bowling, 2009). Del mismo modo, los cuestionarios SODA y WOOP, aunque presentan una alta consistencia interna en sus versiones originales y traducidas, sería recomendable contar con estudios que valoren su aplicabilidad en los servicios de salud sanitaria (Hackert, van Exel \& Brouwer, 2020). 


\section{CONCLUSIÓN}

En síntesis, se encontró gran sustento científico que avala la validez y confiabilidad de los cuestionarios whoqol old, especialmente desarrollado para población adulta mayor y whoqol bref, que es utilizado en población general como una versión corta del test de calidad de vida de la misma Organización Mundial de la Salud; los instrumentos antes mencionados son útiles en pacientes clínicos, institucionalizados, con prevalencia de enfermedades y personas adultas mayores en general; su idioma original es el inglés sin embargo se encuentran validados en una amplia variedad de idiomas y culturas, especialmente personas de habla hispana.

Entre otros hallazgos, los cuestionarios relativamente actuales (opqol-35, soda, woop), se han dedicado a establecer nuevas dimensiones de satisfacción personal que se encuentran relacionados con la calidad de vida, por tal motivo, surgen herramientas que también presentan una alta validez y confiabilidad en sus estudios; sin embargo, se requiere de investigaciones que demuestren su sustentabilidad a lo largo del tiempo y la aplicación en diversos contextos socioculturales y de servicios de salud sanitaria. 


\section{REFERENCIAS BIBLIOGRÁFICAS}

Amonkar, P., Mankar, M. J., Thatkar, P., Sawardekar, P., Goel, R., \& Anjenaya, S. (2018). A Comparative Study of Health Status and Quality of Life of Elderly People Living in Old Age Homes and within Family Setup in Raigad District, Maharashtra. Indian journal of community medicine: official publication of Indian Association of Preventive \& Social Medicine,43(1), 10-13. https://doi.org/10.4103/ijcm.IJCM_301_16

Bangun, B. C. K., Gondodiputro, S., \& Andayani, S. (2020). Insomnia and Quality of Life in The Elderly: WHOQOL-BREF and WHOQOL-OLD Indonesian Version. KEMAS: Jurnal Kesehatan Masyarakat, 16(2). https://www.researchgate.net/publication/348598889/

Bowling, A., \& Rai, G. (2009). Quality of life in healthcare decisions. Medical Ethics and the Elderly, 149-56. https://www.researchgate.net/publication/232726324/

Bowling A. (2009). The Psychometric Properties of the Older People's Quality of Life Questionnaire, Compared with the CASP-19 and the WHOQOL-OLD. Current gerontology and geriatrics research, 2009, 298950. https://doi.org/10.1155/2009/298950

Bowling, A., Hankins, M., Windle, G., Bilotta, C., \& Grant, R. (2013). A short measure of quality of life in older age: the performance of the brief Older People's Quality of Life questionnaire (OPQOLbrief). Archives of gerontology and geriatrics, 56(1), $181-187$. https://doi.org/10.1016/j.archger.2012.08.012

Caliskan, H., Sengul Aycicek, G., Ozsurekci, C., Dogrul, R. T., Balci, C., Sumer, F., Ozcan, M., Karabulut, E., Halil, M., Cankurtaran, M., \& Yavuz, B. B. (2019). Turkish validation of a new scale from older people's perspectives: Older people's quality of life-brief (OPQOL-brief). Archives of gerontology and geriatrics, 83, 91-95. https://doi.org/10.1016/j.archger.2019.04.002

Callaghan, L., Brookes, N., \& Palmer, S. (2017). Older people receiving family-based support in the community: a survey of quality of life among users of 'Shared Lives' in England. Health \& social care in the community, 25(5), 1655-1666. https://doi.org/10.1111/hsc.12422

Casamali, F., Schuch, F. B., Scortegagna, S. A., Legnani, E., \& De Marchi, A. (2019). Accordance and reproducibility of the electronic version of the WHOQOL-BREF and WHOQOL-OLD questionnaires. Experimental gerontology, 125, 110683. https://doi.org/10.1016/j.exger.2019.110683

Chen, Y., \& While, A. E. (2019). Older people living alone in Shanghai: A questionnaire survey of their life experience. Health \& social care in the community, 27(1), 260-269. https://doi.org/10.1111/hsc. 12648

Cheung, Y. B., Yeo, K. K., Chong, K. J., Khoo, E. Y., \& Wee, H. L. (2017). Reliability and validity of the English-, Chinese-and Malay-language versions of the World Health Organization quality of life (WHOQOL-BREF) questionnaire in Singapore. Ann Acad Med Singap, 46(12), 461-9. https://pubmed.ncbi.nlm.nih.gov/29355283/

De Melo, R. L. P., da Silva Júnior, E. G., Souto, R. Q., Leão, Í. S., \& do Carmo Eulálio, M. (2018). Psychometric properties of the complete version of the World Health Organization Quality of Life Assessment (WHOQOL-OLD): reduced response scale. Psicologia: Reflexão e Crítica, 31(1). https://www.researchgate.net/publication/322811322/ 
Espinoza, I., Osorio, P., Torrejón, M. J., Lucas-Carrasco, R., \& Bunout, D. (2011). Validación del cuestionario de calidad de vida (WHOQOL-BREF) en adultos mayores chilenos. Revista médica de Chile, 139(5), 579-586. https://www.researchgate.net/publication/269570814/

Fastame, M. C., Penna, M. P., \& Hitchcott, P. K. (2019). SODA: a new questionnaire for the assessment of life satisfaction in late life span. Aging Clinical and Experimental Research. https://link.springer.com/article/10.1007/s40520-019-01211-9

Feizi, A., \& Heidari, Z. (2020). Persian version of the brief Older People's Quality of Life questionnaire (OPQOL-brief): the evaluation of the psychometric properties. Health and quality of life outcomes, 18(1), 1-11. https://www.researchgate.net/publication/345341165/

Fleck, M. P., Chachamovich, E., \& Trentini, C. (2006). Development and validation of the Portuguese version of the WHOQOL-OLD module. Revista de saude publica, 40(5), 785-791. https://doi.org/10.1590/s0034-89102006000600007

Flores-Herrera, B. I., Castillo-Muraira, Y., Ponce-Martínez, D., Miranda-Posadas, C., Peralta-Cerda, E. G., \& Durán-Badillo, T. (2018). Percepción de los adultos mayores acerca de su calidad de vida. Una perspectiva desde el contexto familiar. Revista de Enfermería del Instituto Mexicano del Seguro Social, 26(2), 83-88. https://www.researchgate.net/publication/326753254/

Gobbens, R. J. J., \& van Assen, M. A. L. M. (2016). Psychometric properties of the Dutch WHOQOLOLD. Health and Quality of Life Outcomes, 14(1). https://doi:10.1186/s12955-016-0508-5

Hackert, M., Brouwer, W., Hoefman, R. J., \& van Exel, J. (2019). Views of older people in the Netherlands on wellbeing: A Q-methodology study. Social science \& medicine (1982), 240, 112535. https://doi.org/10.1016/j.socscimed.2019.112535

Hackert, M., van Exel, J., \& Brouwer, W. (2020). Well-being of Older People (WOOP): Quantitative validation of a new outcome measure for use in economic evaluations. Social science \& medicine (1982), 259, 113109. https://doi.org/10.1016/j.socscimed.2020.113109

Haugan, G., Drageset, J., André, B., Kukulu, K., Mugisha, J., \& Utvær, B. (2020). Assessing quality of life in older adults: psychometric properties of the OPQoL-brief questionnaire in a nursing home population. Health and quality of life outcomes, 18(1), 1. https://doi.org/10.1186/s12955-019-1245-3

Hernández-Navor, J. C., Guadarrama-Guadarrama, R., Castillo-Arrellano, S. S., Hernández, G. A., \& Márquez-Mendoza, O. (2015). Validación del WHOQOL-OLD en adultos mayores de México. PSIENCIA. Revista Latinoamericana de Ciencia Psicológica, 7(3), 397-405. https://www.redalyc.org/pdf/3331/333143435001.pdf

Huerta, J. A. L., Romo, R. A. G., \& Tayabas, J. M. T. (2017). Propiedades psicométricas de la versión en español de la Escala de Calidad de Vida WHO QoL BREF en una muestra de adultos mexicanos. Rev Iberoam Diag Eval-E Aval $\quad$ Psicológica, 2(44), $\quad 105-15$. https://www.researchgate.net/publication/318082767/

Hussenoeder, F. S., Conrad, I., Roehr, S., Fuchs, A., Pentzek, M., Bickel, H., \& Mamone, S. (2020). Mild cognitive impairment and quality of life in the oldest old: a closer look. Quality of Life Research, 1-9. https://link.springer.com/article/10.1007/s11136-020-02425-5 
Kalfoss, M. H., Reidunsdatter, R. J., Klöckner, C. A., \& Nilsen, M. (2021). Validation of the WHOQOLBref: psychometric properties and normative data for the Norwegian general population. Health and quality of life outcomes, 19(1), 13. https://doi.org/10.1186/s12955-020-01656-X

Kim, H. Y., Nho, J. H., Kim, J. Y., \& Kim, S. R. (2021). Validity and reliability of the Korean version of the world health organization quality of life instrument-older adults module. Geriatric nursing (New York, N.Y.), 42(2), 548-554. https://doi.org/10.1016/j.gerinurse.2020.10.006

Kruithof, N., Haagsma, J. A., Karabatzakis, M., Cnossen, M. C., de Munter, L., van de Ree, C., de Jongh, M., \& Polinder, S. (2018). Validation and reliability of the Abbreviated World Health Organization Quality of Life Instrument (WHOQOL-BREF) in the hospitalized trauma population. Injury, 49(10), 1796-1804. https://doi.org/10.1016/j.injury.2018.08.016

Leles da Costa Dias, F., Teixeira, A. L., Cerqueira Guimarães, H., Borges Santos, A. P., Rios Fonseca Ritter, S., Barbosa Machado, J. C., Tonidandel Barbosa, M., \& Caramelli, P. (2019). Prevalence of latelife depression and its correlates in a community-dwelling low-educated population aged 75+ years: The Pietà study. Journal of affective disorders, 242, 173-179. https://doi.org/10.1016/j.jad.2018.08.012

Lin, C. Y., Hwang, J. S., Wang, W. C., Lai, W. W., Su, W. C., Wu, T. Y., Yao, G., \& Wang, J. D. (2019). Psychometric evaluation of the WHOQOL-BREF, Taiwan version, across five kinds of Taiwanese cancer survivors: Rasch analysis and confirmatory factor analysis. Journal of the Formosan Medical Association = Taiwan yi zhi, 118(1 Pt 2), 215-222. https://doi.org/10.1016/j.jfma.2018.03.018

Lucas-Carrasco, R. (1998). Versión Española del WHOQOL. Editorial Ergón. Madrid.https://www.sspa.juntadeandalucia.es/servicioandaluzdesalud/sites/default/files/sincfiles/wsasmedia-pdf_publicacion/2020/18-WHOQOL-BREF.pdf

Lucas-Carrasco, R., Laidlaw, K., \& Power, M. J. (2011). Suitability of the WHOQOL-BREF and WHOQOL-OLD for Spanish older adults. Aging \& mental health, 15(5), 595-604. https://doi.org/10.1080/13607863.2010.548054

Machón, M., Larrañaga, I., Dorronsoro, M., Vrotsou, K., \& Vergara, I. (2017). Health-related quality of life and associated factors in functionally independent older people. BMC geriatrics, 17(1), 19. https://doi.org/10.1186/s12877-016-0410-3

Mares, J., Cigler, H., \& Vachkova, E. (2016). Czech version of OPQOL-35 questionnaire: the evaluation of the psychometric properties. Health and quality of life outcomes, 14(1), 1-13. https://www.researchgate.net/publication/304070552/

Martín-María, N., Lara, E., Cresswell-Smith, J., Forsman, A. K., Kalseth, J., Donisi, V., Amaddeo, F., Wahlbeck, K., \& Miret, M. (2021). Instruments to evaluate mental well-being in old age: a systematic review. Aging \& mental health, 25(7), 1191-1205. https://doi.org/10.1080/13607863.2020.1774742

Nikkhah, M., Heravi-Karimooi, M., Montazeri, A., Rejeh, N., \& Sharif Nia, H. (2018). Psychometric properties the Iranian version of Older People's Quality Of Life questionnaire (OPQOL). Health and quality of life outcomes, 16(1), 174. https://doi.org/10.1186/s12955-018-1002-z

Nuntaboot, K., Boonsawasdgulchai, P., \& Bubpa, N. (2019). Roles of mutual help of local community networks in community health activities: Improvement for the quality of life of older people in Thailand. International Journal of Nursing Sciences. https://doi.org/10.1016/j.ijnss.2019.04.001 
Orellana, K., Manthorpe, J., \& Tinker, A. (2020). Day centres for older people: a systematically conducted scoping review of literature about their benefits, purposes and how they are perceived. Ageing and society, 40(1), 73-104. https://doi.org/10.1017/s0144686x18000843

Perera, H. N., Izadikhah, Z., O'Connor, P., \& McIlveen, P. (2018). Resolving Dimensionality Problems With WHOQOL-BREF Item Responses. Assessment, 25(8), 1014-1025. https://doi.org/10.1177/1073191116678925

Power, M., Quinn, K., Schmidt, S., \& WHOQOL-OLD Group (2005). Development of the WHOQOLold module. Quality of life research: an international journal of quality of life aspects of treatment, care and rehabilitation, 14(10), 2197-2214. https://doi.org/10.1007/s11136-005-7380-9

Power, M., Harper, A., \& Bullinger, M. (1999). The World Health Organization WHOQOL-100: tests of the universality of Quality of Life in 15 different cultural groups worldwide. Health psychology: official journal of the Division of Health Psychology, American Psychological Association, 18(5), 495-505. https://doi.org/10.1037//0278-6133.18.5.495

Quiroz, C. O. A., García, J. J. V., Castro, S. B. E., Encinas, D. M. S., \& Flores, R. G. (2013). Confiabilidad y validez del Cuestionario de Calidad de Vida (WHOQOL-OLD) en adultos mayores mexicanos. Psicología y salud, 23(2), 241-250. https://www.researchgate.net/publication/308634637

Reba, K., Birhane, B. W., \& Gutema, H. (2019). Validity and Reliability of the Amharic Version of the World Health Organization's Quality of Life Questionnaire (WHOQOL-BREF) in Patients with Diagnosed Type 2 Diabetes in Felege Hiwot Referral Hospital, Ethiopia. Journal of diabetes research, 2019, 3513159. https://doi.org/10.1155/2019/3513159

Reyes Rodríguez, E., \& Durand Rill, R. (2018). Quality of life in the elderly from the University of the Elderly. Revista Información Científica, 97(1), 192-204. https://doi.org/10.3844/ajassp.2012.71.74

Rezaeipandari, H., Morowatisharifabad, M. A., Mohammadpoorasl, A., \& Shaghaghi, A. (2020). Crosscultural adaptation and psychometric validation of the World Health Organization quality of life-old module (WHOQOL-OLD) for Persian-speaking populations. Health and quality of life outcomes, 18(1), 1-7. https://doi.org/10.1186/s12955-020-01316-0

Robles, A. R. (2017). Calidad de vida y polifarmacia del adulto mayor integrante del programa "adultos mayores empacadores". NURE investigación: Revista Científica de enfermería, 14(91), 5. https://www.nureinvestigacion.es//OJS/index.php/nure/article/view/1206

Rosén, H., Ahlström, G., \& Lexén, A. (2020). Psychometric properties of the WHOQOL-BREF among next of kin to older persons in nursing homes. Health and quality of life outcomes, 18(1), 103. https://doi.org/10.1186/s12955-020-01345-9

Samy, A. L., Kamaruzzaman, S. B., Krishnaswamy, S., \& Low, W. Y. (2020). Predictors of Quality of Life among Older People with Mild Cognitive Impairment Attending Urban Primary Care Clinics. Clinical gerontologist, 43(4), 441-454. https://doi.org/10.1080/07317115.2019.1608611

Saqib Lodhi, F., Raza, O., Montazeri, A., Nedjat, S., Yaseri, M., \& Holakouie-Naieni, K. (2017). Psychometric properties of the Urdu version of the World Health Organization's quality of life questionnaire (WHOQOL-BREF). Medical journal of the Islamic Republic of Iran, 31, 129. https://doi.org/10.14196/mjiri.31.129 
Simeão, S., Martins, G., Gatti, M., Conti, M., Vitta, A., \& Marta, S. N. (2018). Comparative study of quality of life of elderly nursing home residents and those attneding a day Center. Estudo comparativo da qualidade de vida de idosos asilados e frequentadores do centro dia. Ciencia \& saude coletiva, 23(11), 3923-3934. https://doi.org/10.1590/1413-812320182311.21742016

Siriwardhana, D. D., Weerasinghe, M. C., Rait, G., Scholes, S., \& Walters, K. R. (2019). The association between frailty and quality of life among rural community-dwelling older adults in Kegalle district of Sri Lanka: a cross-sectional study. Quality of life research : an international journal of quality of life aspects of treatment, care and rehabilitation, 28(8), 2057-2068. https://doi.org/10.1007/s11136-019-02137-5

Snell, D. L., Siegert, R. J., Surgenor, L. J., Dunn, J. A., \& Hooper, G. J. (2016). Evaluating quality of life outcomes following joint replacement: psychometric evaluation of a short form of the WHOQOLBref. Quality of life research: an international journal of quality of life aspects of treatment, care and rehabilitation, 25(1), 51-61. https://doi.org/10.1007/s11136-015-1044-1

Suárez, L., Tay, B., \& Abdullah, F. (2018). Psychometric properties of the World Health Organization WHOQOL-BREF Quality of Life assessment in Singapore. Quality of life research: an international journal of quality of life aspects of treatment, care and rehabilitation,27(11), 2945-2952. https://doi.org/10.1007/s11136-018-1947-8

Tavares, D. M., Matias, T. G., Ferreira, P. C., Pegorari, M. S., Nascimento, J. S., \& Paiva, M. M. (2016). Quality of life and self-esteem among the elderly in the community. Qualidade de vida e autoestima de idosos na comunidade. Ciencia \& saude coletiva, 21(11), 3557-3564. https://doi.org/10.1590/1413812320152111.03032016

Varela, L. F. (2016) Salud y calidad de vida en el adulto mayor. Rev Peru Med Exp Salud Publica, 33(2):199-201. http://dx.doi.org/10.17843/rpmesp.2016.332.2196

Vilar, M., Sousa, L. B., \& Simões, M. R. (2016). The European Portuguese WHOQOL-OLD module and the new facet Family/Family life: reliability and validity studies. Quality of life research: an international journal of quality of life aspects of treatment, care and rehabilitation, 25(9), 2367-2372. https://doi.org/10.1007/s11136-016-1275-9

World Health Organization. (2015). People-centred and integrated health services: an overview of the evidence: interim report (No. WHO/HIS/SDS/2015.7). World Health Organization. https://apps.who.int/iris/bitstream/handle/10665/155004/WHO_HIS_SDS_2015.7_eng.pdf?sequence=1 \&isAllowed $=y$ 